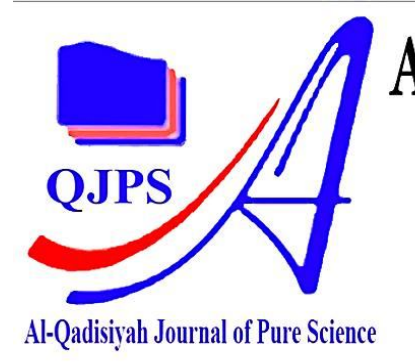

Al-Qadisiyah Journal of Pure Science

ISSN(Printed): 1997-2490

ISSN(Online):

$2411-3514$

DOI : /10.29350/jops.

http://qu.edu.iq/journalsc/index.php/JOPS

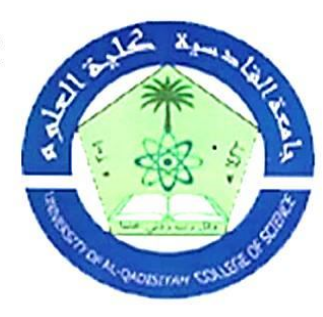

\title{
Optical Patterns Controlled by Computerized Spatial Light Modulator
}

\section{Authors Names \\ Hayder. A. Naser \\ Article History \\ Received on: 02 / 03 / 2020 \\ Revised on: 18/ 04/2020 \\ Accepted on: 05 / 05 / 2020}

Keywords:

Spatial Light Modulator (SLM), Liquid crystal (LC), Brightness, Contrast, diffraction patterns.

DOI: https://doi.org/10.29350/ jops.2020.25. 2.1075

\section{ABSTRACT}

In this work, a contemporary optical system designed using a technology of spatial light modulator (SLM). This system is depending on the ability of altering light beam into different optical patterns according to a signal feedback injected computationally to the SLM. The effects of control parameters on the SLM operation are studied such us brightness (B) which determines the constant DC voltage applied to SLM pixels, and contrast (C) determines the maximum AC voltage amount that can applied to the SLM. The influences of the transmission linearity of image intensity controlled by the gamma adjustment, which can used to align non-linearity of image intensity. The grey scale used to control and imagine which signals corresponding to grey levels and image location. The resolution and orientation of the obtained images altered by the screen format to make optical experiments more satisfied, hence different ratios of amplitude and phase modulation can understood.

MSC: 30C45, $30 \mathrm{C50}$

\section{Introduction}

A spatial light modulator (SLM) is an electrically programmable expedient that moderates light rendering to an immovable spatial (pixel) position. SLMs characteristically used to regulate incident light in amplitude-only, phase-only or the both (phase-amplitude). They are contemporary holographic plans built with liquid crystal (LC) displays, which form the received light field by adjusting its phase, its amplitude, or both, as a function of location on the arrangement [1]. These SLMs are have a tendency to achieve phase-only inflection deprived of momentous intensity reduction [2]. SLM technologies have developed along with the creation of practical application such us holographic imaging [3], Boulder Nonlinear Systems [4], high and low two-level driver voltage circuit [4] and in the spatial implementation of reservoir computing (6). The knowledge of SLM raised from the necessity to rapidly translate data in electrical form into spatially moderated intelligible optical signs. This permits for the ability to present data into an optical system, since the information could approved straight by the optical amplitude. To 
accomplish this, one requirement is to operate the multifaceted optical fields conveyed through the optical system. Initially photographic pictures used for wave front variation but abundant more influential optical information dispensation systems could be understood if the SLM is substituted to accomplish of altering transited light in real time [7]. The SLM is involuntary as a sinusoidal grating with revolving alignment and/ or altering retro. The temporally altered diffraction light beams used to irradiate a motionless object [8]. Hence the LC SLM, which is a real time electro-optic tool, proficient of moderating the optical characteristics of an optical wave front in space and time [9] . It represents an adaptive tool in optics combined in various optical systems and it provides a regulatory improvement of the production of these systems [10]. The effect of functional voltage presents alteration sign; this inflection modifies phase behavior, with alterations in LC response dependent on the way of SLM connection [11]. The technique of the SLM produce decreasing in the optical deviations, which produce an excellence with a SLM[12].

The spiral construction of the twisted nematic crystal is used to alteration the polarization of light. Once the polarization of the light is parallel to the particles of the cell at the arrival surface, the polarization tracks the rotation of the particle axis. Therefore, the light will have a polarization vertical to the incident polarization. In order to understand an active optical component, a voltage applied to the LC cell, Figure 2. The LC mesh offers an electrically controlling of light.

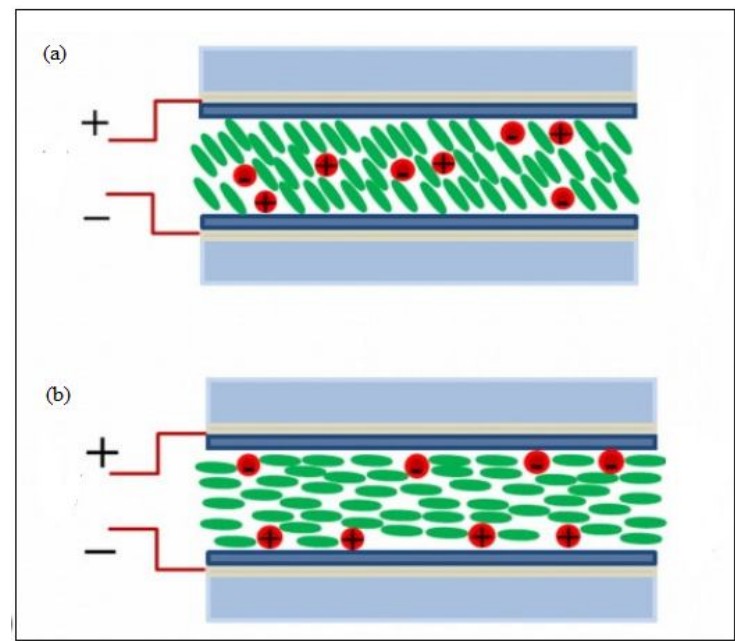

Figure2. Effects of exterior voltage exerts on the twisted nematic LC. (a) V = zero, with twisted (c) the particles arrangement according to the applied voltage applied.

The SLM procedure of phase or amplitude inflection is contingent on the LC possessions of "birefringence". The LC pixels are preparation in approximately way that the alignment of respectively pixel is dependent on the electric field. The production forms permit the discrete optical recompense of each phase and amplitude. The various refractive indices present a joint 
phase delay between the two partials fields consistent to the two linear polarizations, Figure 3[13-15]. The shift of the minima $\Delta y$ and the length of the period g [16] give the phase delay "shift" $\Delta \Phi$ between two grey levels.

$$
\Delta \Phi=\frac{2 \pi}{g} . \Delta y
$$

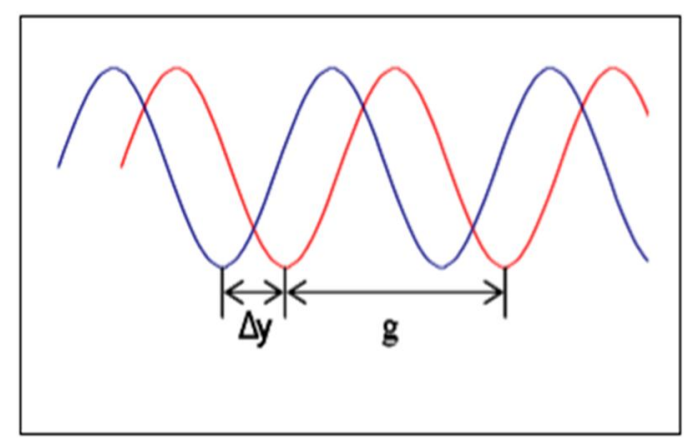

Figure 3. Alteration of the interfering minima produce altering in phase.

When a voltage applied to the cell, the molecules make align parallel to the electric field. This will produce a reducing in anisotropy of the LC because of the angle between the path of light spread and the molecular axes becomes slighter pending finally together directions are parallel, and the optical axis of the LC is parallel to the direction of light propagation. For this condition the Jones matrix can approached as: [17]

$$
W_{T N-L C} \approx R(-\alpha)\left(\begin{array}{cc}
\cos \alpha & \sin \alpha \\
-\sin \alpha & \cos \alpha
\end{array}\right)=\left(\begin{array}{ll}
1 & 0 \\
0 & 1
\end{array}\right)
$$

Where $R(-\alpha)$ represents a half-wave plate with an optical axis inclined by an angle $\delta$ with respect to the $\mathrm{x}$ direction of the coordinate system. This equation shows that when the applied voltage to the LC cell is large, there will not changing in light polarization. As a result, the incident light with linear polarization leaves the cell with an elliptic state of polarization. Typically, SLMs inserted between two polarizers in order to adjust its multifaceted transmittance, the transmission occupation is:

$$
T\left(\emptyset_{D}, \emptyset, \beta ; \Psi_{1}, \Psi_{2}\right)=J_{P}\left(\Psi_{2}\right) J_{L C D}\left(\emptyset_{D}, \varnothing, \beta\right) J_{P}\left(\Psi_{1}\right)
$$

where $\mathrm{J}_{\mathrm{P}}$ and $\mathrm{J}_{\mathrm{LCD}}=$ the Jones matrices of polarizer and LC-SLM, respectively.

$\emptyset_{D}=$ the azimuth angle of LC-SLM front director.

$\varnothing=$ the twist angle.

$\beta=$ the birefringence;

$\Psi_{1}$ and $\Psi_{2}=$ the azimuth angles of extraordinary polarizer axes [14]. Writing Eq. (1) in its complex form: 


$$
T(x)=|T(x)| \exp i \delta(x)
$$

where $\mathrm{T}(\mathrm{x})$ and $\delta(\mathrm{x})=$ the amplitude transmittance and phase transmittance; respectively [18].

\section{Experimental Setup}

The system consists of polarizer, SLM, analyzer, and a He-Ne laser operating at $632.8 \mathrm{~nm}$ wavelength and power of $5 \mathrm{~W}$, the photograph of the experimental setup shown in Figure 4 . The SLM device is a Sony LCX016AL-6 type with active area of $26.6 \mathrm{~mm} \times 20 \mathrm{~mm}$, operating at 5V and the number of image pixels are $832 \times 624$. The SLM control program uses RS-232 instructions to make its errands on the LCD. This makes it probable to integrate the SLM in original systems. The overloaded picture will altered to a picture with 256 grey-scale standards. The two polarizers with any rotation used to achieve the controlling of the system transmission. The obtained images focused into CCD by a lens of $10 \mathrm{~cm}$.

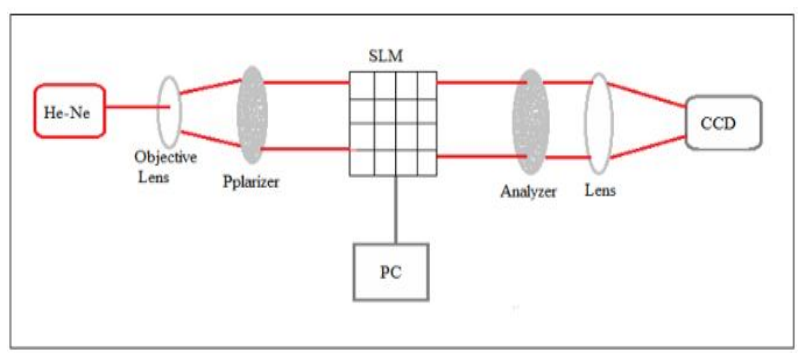

Figure 4. The experimental setup.

The applied voltages can controlled by using lens phase and prism phase tools, Fig. 5. Drive board receives this obtained information as grey level standards of the sign formed by the VGA output of a photograph's connecter of a mutual PC.

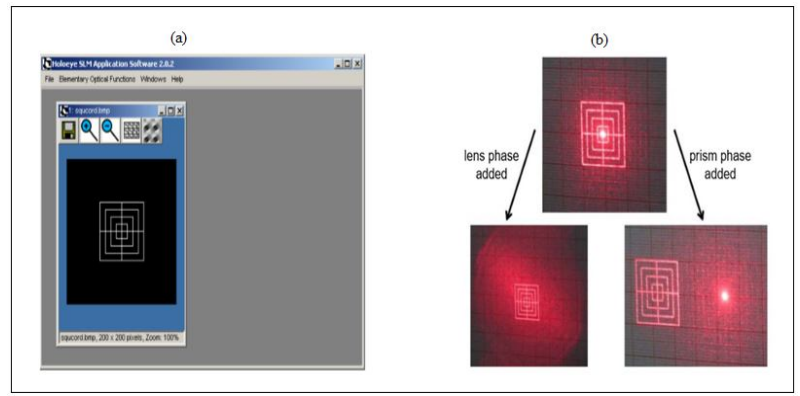

Figure 5. (a) Image space of the SLM application software, (b) controlling the images by the lens phase and prism phase tools. 
The resulting forms represent images created by the controlling optical elements (which often denoted to as gratings), display a distinct far-field deflection forms of the spatially intermittent items. This is due to spatial occurrence field of the sporadic substances that involves distinct rates only if the spreading items dynamically shaped with the SLM. Figure (6) shows the obtained results for certain diffracting objects. All diffraction angles produced by the signal on the LC display are controlled. They related to electrical signals controlling the SLM; an AC voltage applied to each individual pixel in the SLM. The experimental diffraction patterns show convinced symmetries, it is noticeable that rotationally symmetrical objects form a rotationally symmetrical diffraction patterns. Select the evolution arguments can no lengthier wholly easily when the gratings produced with the support of the dynamic addressable SLM. The effects of diffraction appear when the SLM is addressed, figure 6(a-i), these are gained when the sample points are limited in its spatial frequencies, and the obtained amplitudes are reappearances of the controlled essential field. The voltages that are functional to the SLM allowed a practically continuous evolution of the 'spiral state' that alternates the incident polarization to the isotropic state.

(a)

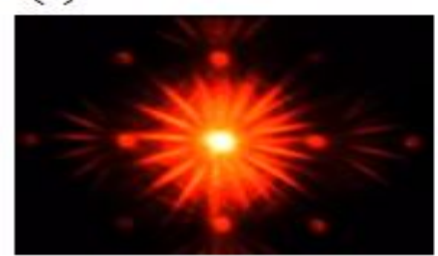

(d)

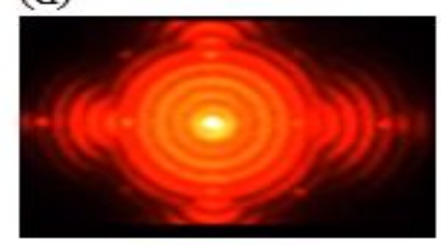

(g)

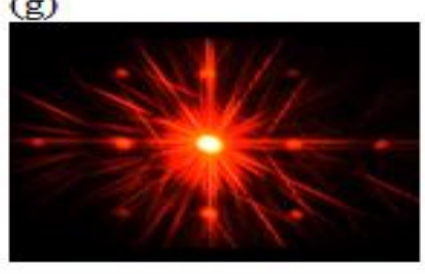

(b)

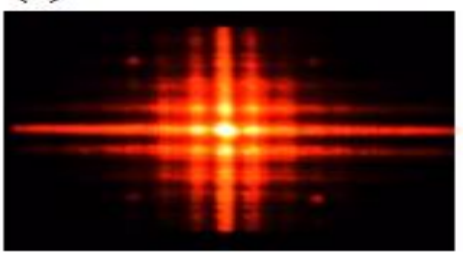

(e)

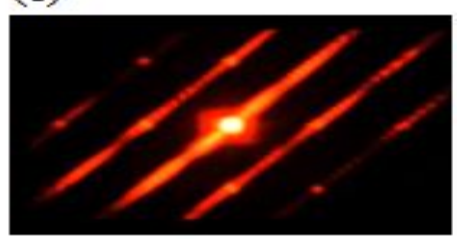

(h)

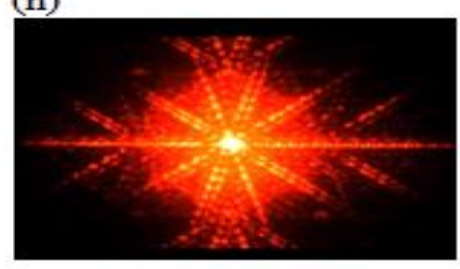

(c)

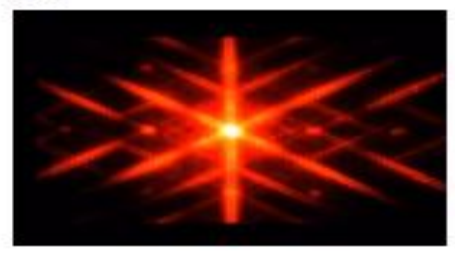

(f)

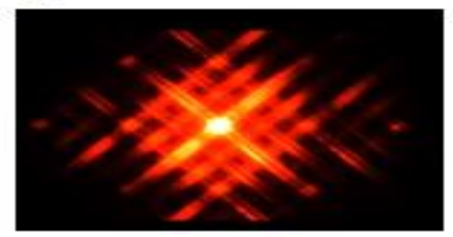

(i)

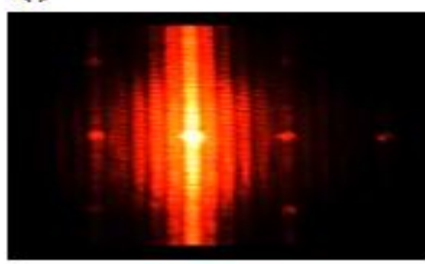

Figure 6 (a-i). Influence of controlling SLM on the incident He-Ne laser beam, the different patterns are a result of different control parameters. The symmetric in the diffraction patterns with respect to the $\mathrm{x}$ - or the $\mathrm{y}$-axis are shown.

Because of the SLM modulating, a new wave obtained, in which the phase changes in a random way, but in the same way. The difference $\left(\delta_{1}-\delta_{2}\right)$ then remains constant. Later paths of different length, producing a phase difference, the limited waves will reunited. The transmission of SLM between two polarizers is function of polarizer's angles $\Psi_{1}$ and $\Psi_{2}$, gray level of incident signal, 
brightness B, contrast C, and Geometry (image width, position, and sharpness control) all improved mechanically. Table (1) illustrates the values of these control parameters for each optical pattern generated by SLM and showed in figure (6).

Table 1. Formation numbers of the control limits inserted to the SLMs control program.

\begin{tabular}{|l|l|l|l|l|l|l|}
\hline $\begin{array}{l}\text { Image } \\
\text { number }\end{array}$ & $\begin{array}{l}\text { Phase } \\
\text { shift } \\
\Delta \Phi\end{array}$ & $\begin{array}{l}\text { Grey } \\
\text { level }\end{array}$ & $\begin{array}{l}\text { Lens } \\
\text { phase }\end{array}$ & $\begin{array}{l}\text { Prism } \\
\text { phase }\end{array}$ & $\begin{array}{l}\text { Contrast } \\
\text { C }\end{array}$ & $\begin{array}{l}\text { Brightness } \\
\text { B }\end{array}$ \\
\hline a & 2 & 0 & 4 & 5 & 195 & 100 \\
\hline b & 4 & 1 & 7 & 10 & 179 & 112 \\
\hline c & 6 & 2 & 9 & 13 & 221 & 150 \\
\hline d & 8 & 3 & 14 & 22 & 119 & 122 \\
\hline e & 10 & 4 & 22 & 24 & 100 & 99 \\
\hline f & 12 & 5 & 23 & 27 & 222 & 199 \\
\hline g & 14 & 6 & 25 & 29 & 200 & 189 \\
\hline h & 16 & 7 & 33 & 36 & 225 & 200 \\
\hline i & 18 & 8 & 45 & 43 & 190 & 195 \\
\hline
\end{tabular}

\section{Conclusion}

A programmable SLM with visual apparatuses investigated to present variety of optical patterns of the incident laser beam, where the SLM operate as a sinusoidal grating with revolving alignment in different retro. The Different speckles, which formed by this system, and the control parameters, achieved the image superiority such as lens, prism, contrast and brightness are studied. We have demonstrated that the SLM device exhibits a solution for low cost and direct applications benefits with low power ingesting as a substitute of traditional multi- lens systems for mutable focal length with a compound motorized operation. A practical performance in imaging systems attained utilizing SLM, which can modify optical imaging because of its driving ability. The LC inserted between polarizers can therefore be used not only as an image basis in a prediction system, but also (with additional polarizer locations) as a switchable diffractive component which can be used to signify dynamically optical elements like: Axicon gray level image, cylindrical Fresnel zone lens, and vortex phase image, all these are created with a 256gray level. 


\section{References}

[1] Nikolenko, V., Peterka, D. S., \& Yuste, R. (2010). A portable laser photostimulation and imaging microscope. Journal of neural engineering, 7(4), 045001.

[2] Nguyen, T. H., \& Popescu, G. (2013). Spatial Light Interference Microscopy (SLIM) using twisted-nematic liquid-crystal modulation. Biomedical optics express, 4(9), 1571-1583.

[3] Hyde, S. C. W., Barry, N. P., Jones, R., Dainty, J. C., \& French, P. M. W. (1996). High resolution depth resolved imaging through scattering media using time resolved holography. Optics communications, 122(4-6), 111-116.

[4] Harriman, J., Gauza, S., Wu, S. T., Wick, D., Bagwell, B., Martinez, T., ... \& Serati, S. (2006, February). Transmissive spatial light modulators with high figure-of-merit liquid crystals for foveated imaging applications. In Liquid Crystal Materials, Devices, and Applications XI (Vol. 6135, p. 61350C). International Society for Optics and Photonics.

[5] Wu, L., Zhou, F. N., \& Zhu, M. (2016). A Driver Circuit of Spatial Light Modulator. International Journal of Future Generation Communication and Networking, 9(6), 255-262.

[6] Kitayama, K. I., Notomi, M., Naruse, M., Inoue, K., Kawakami, S., \& Uchida, A. (2019). Novel frontier of photonics for data processing — Photonic accelerator. APL Photonics, 4(9), 090901.

[7] Goodman, J. W. (2005). Introduction to Fourier optics. Roberts and Company Publishers.

[8] Egge, S. V., Welde, K., Oesterberg, U. L., Aksnes, A., Akram, N. M., Kartashov, V., \& Tong, Z. (2011). Sinusoidal rotating grating for speckle reduction in laser projectors: feasibility study. Optical Engineering, 50(8), 083202.

[9] Solodar, A., Klapp, I., \& Abdulhalim, I. (2014). Annular liquid crystal spatial light modulator for beam shaping and extended depth of focus. Optics Communications, 323, 167-173.

[10] Bondareva, A. P., Cheremkhin, P. A., Evtikhiev, N. N., Krasnov, V. V., Starikov, R. S., \& Starikov, S. N. (2014). Measurement of characteristics and phase modulation accuracy increase of LC SLM" HoloEye PLUTO VIS". In Journal of Physics: Conference Series (Vol. 536, No. 1, p. 012011). IOP Publishing.

[11] Farré, A., Shayegan, M., López-Quesada, C., Blab, G. A., Montes-Usategui, M., Forde, N. R., \& Martín-Badosa, E. (2011). Positional stability of holographic optical traps. Optics express, 19(22), 21370-21384.

[12] Jesacher, A., Schwaighofer, A., Fürhapter, S., Maurer, C., Bernet, S., \& Ritsch-Marte, M. (2007). Wavefront correction of spatial light modulators using an optical vortex image.Optics express, 15(9), 5801-5808.

[13] Hecht, M. L. (1978). Hecht. Michael L. Measures of communication satisfaction, Human Communication research, 4(4), 350.

[14] Turunen, J., \& Wyrowski, F. (1998). Diffractive optics for industrial and commercial applications. Wiley-VCH, 440. 
[15] Kress, B., \& Meyrueis, P. (2000). Digital diffractive optics. John Wiley\&Sons Ltd.

[16] Morrison, R. L. (1992). Symmetries that simplify the design of spot array phase gratings. JOSA A, 9(3), 464-471.

[17] Yamauchi, M. (2005). Jones-matrix models for twisted-nematic liquid-crystal devices. Applied optics, 44(21), 4484-4493.

[18] Rickenstorff, C., \& Ostrovsky, A. S. (2010). Measurement of the amplitude and phase modulation of a liquid crystal spatial light modulator. Superficies y vacío, 23, 36-39. 